\title{
Electronic band gaps of confined linear carbon chains ranging from polyyne to carbyne
}

\author{
Lei Shi, ${ }_{1}^{1}$ Philip Rohringer, ${ }^{1,2}$ Marius Wanko, ${ }^{3}$ Angel Rubio, ${ }^{3,4}$ Sören Waßerroth, ${ }^{5}$ Stephanie Reich, ${ }^{5}$ Sofie Cambré, ${ }^{2}$ \\ Wim Wenseleers, ${ }^{2}$ Paola Ayala, ${ }^{1}$ and Thomas Pichler ${ }^{1, *}$ \\ ${ }^{1}$ Faculty of Physics, University of Vienna, 1090 Wien, Austria \\ ${ }^{2}$ Experimental Condensed Matter Physics Laboratory, University of Antwerp, B-2610 Antwerp, Belgium \\ ${ }^{3}$ Nano-Bio Spectroscopy Group and ETSF, Dpto. Material Physics, Universidad del País Vasco, 20018 San Sebastián, Spain \\ ${ }^{4}$ Max Planck Institute for the Structure and Dynamics of Matter, Hamburg, Germany \\ ${ }^{5}$ Department of Physics, Freie Universität Berlin, Arnimallee 14, 14195 Berlin, Germany
}

(Received 15 March 2017; published 12 December 2017)

\begin{abstract}
Ultralong linear carbon chains of more than 6000 carbon atoms have recently been synthesized within doublewalled carbon nanotubes (DWCNTs), and they show a promising route to one-atom-wide semiconductors with a direct band gap. Theoretical studies predicted that this band gap can be tuned by the length of the chains, the end groups, and their interactions with the environment. However, different density functionals lead to very different values of the band gap of infinitely long carbyne. In this work, we applied resonant Raman excitation spectroscopy with more than 50 laser wavelengths to determine the band gap of long carbon chains encapsulated inside DWCNTs. The experimentally determined band gaps ranging from 2.253 to $1.848 \mathrm{eV}$ follow a linear relation with Raman frequency. This lower bound is the smallest band gap of linear carbon chains observed so far. The comparison with experimental data obtained for short chains in gas phase or in solution demonstrates the effect of the DWCNT encapsulation, leading to an essential downshift of the band gap. This is explained by the interaction between the carbon chain and the host tube, which greatly modifies the chain's bond-length alternation.
\end{abstract}

DOI: 10.1103/PhysRevMaterials.1.075601

\section{INTRODUCTION}

One-dimensional linear carbon chains (LCCs) possess unique properties, one of which is a direct band gap that is tuneable by the length of the chains [1-3]. Despite their apparently simple structure, the properties of long LCCs (LLCCs) are hard to calculate because long-range electron exchange and correlation effects lead to a Peierls distortion, yielding a structure with significant bond-length alternation (BLA) (which, moreover, depends strongly on end-capping effects). Their experimental observation has long remained elusive because of the high reactivity of the chains. Until recently, only relatively short chains (polyynes up to 44 carbon atoms) could be synthesized and stabilized [4], showing a linear relation between band gap and inverse chain length [5]. Being only one atom wide, the electronic spectrum of these short LCCs furthermore depend drastically on the local environment and on the different end groups that are implemented to stabilize the chains, thus providing a whole range of tools to continuously tune the band gap of these materials in a very wide range through length, environment, and end groups [5]. Very recently, we succeeded in stabilizing ultralong LCCs of more than 6000 carbon atoms within double-walled carbon nanotubes (DWCNTs) [6], which now allows us to determine also the electronic band gap of such ultralong chains.

Tuning of the band gap by changing the material's properties plays a crucial role in the design of new semiconductor devices. In the past, fundamental research has focused mainly on other (quasi) one-dimensional systems, in particular on carbon nanotubes, which show a band gap that is strongly dependent on their chiral structure [7]. In addition, the band

*thomas.pichler@univie.ac.at gap of various $2 \mathrm{D}$ materials can also be tuned by the number of layers of the 2D materials [8-12]. Although the range and variability of the band gaps of these (quasi) 1D and 2D systems are huge, as are their possible applications, their band gap is not continuously tuneable within a very wide range, and it varies from direct to indirect band gap by increasing the number of layers. Hence, a material with a tuneable direct band gap is highly desired, for which LCCs with alternating single and triple bonds are a perfect candidate [1].

The band gap for infinite LCCs in vacuum is calculated, and a wide variety of values ranging from 0.2 to $8.5 \mathrm{eV}$ have been reported [3,13]. Intrinsically, the band gap of an LCC depends on the BLA. Therefore, the large variety of predicted band gaps for infinite LCCs can be explained by the difficulty of predicting the BLA of polyynes with density functionals that need to take into account both the electron-phonon coupling and many-electron interactions. The BLA decreases along with the increasing length of the LCC, which changes the electronic structure and results in a smaller band gap. However, this modulation by size has a fundamental limitation because a vanishing BLA can never be reached due to the Peierls distortion [14], which means that the single-triple bonds can never be converted into double-double bonds. Thus, a finite band gap due to the saturation is expected for the infinite LCC (carbyne).

Experimentally, free-standing LCCs need to be end-capped with hydrogen, adamantyl, trityl, tri-isopropylsilyl, or any of many other chemical groups to stabilize them. The longest end-capped LCCs synthesized so far consisted of 44 carbon atoms, with the band gap ranging from 4.7 to $2.6 \mathrm{eV}$ corresponding to lengths of 4-44 carbon atoms, respectively $[4,5,15]$. For LCCs synthesized inside single-walled carbon nanotubes (SWCNTs), the resonance energies of LCCs with different lengths were reported to be $2.6-2.0 \mathrm{eV}$ and were 
assigned to the dipole-forbidden transitions that become active via symmetry breaking by the CNT encapsulation [16-20].

Although the band gap of short LCCs has been well studied, the gap of LLCCs toward carbyne remains elusive, as they are extremely unstable. In this work, we present experimental measurements of the band gaps of LLCCs stabilized within DWCNTs using resonant Raman excitation spectroscopy. Band gaps in the range of 2.253-1.848 eV have been observed. In addition, the feasibility of using long chains has allowed us to determine the smallest band gap of confined carbyne reported so far.

\section{EXPERIMENTS AND METHODS}

The LLCCs used in this study were synthesized inside DWCNTs with narrow inner diameters with a length ranging from about 30 carbon atoms (observed by transmission electron microscopy) up to more than 6000 carbon atoms (confirmed from near-field Raman spectroscopy) as described previously [6]. High-resolution transmission electron microscopy (HRTEM) was performed on a JEOL 2010F microscope conducted at $120 \mathrm{kV}$ to avoid the LLCC decomposition. As shown in Fig. 1, the HRTEM image and the corresponding simulations clearly confirm the hybrid structure of LLCC@DWCNT as the line profile consists of five peaks corresponding to two walls of a DWCNT and a LLCC in the middle. This unambiguously proves that the middle line represents a real LLCC and not a ghost contrast [21]. For this hybrid system with a LLCC longer than $10 \mathrm{~nm}$ (i.e., more than 80 carbon atoms), the distance between the tubes is almost the same as that to the LLCC, suggesting similar interactions. These interactions have to be considered for proper analysis of the following Raman spectra of LLCC@DWCNTs and the band gaps of LLCCs [22].

Unfortunately, the band gaps for LLCCs@DWCNTs cannot be investigated directly by absorption spectroscopy, as was done for the end-capped short LCCs, because the weak signal from the LLCCs is completely overlapped and

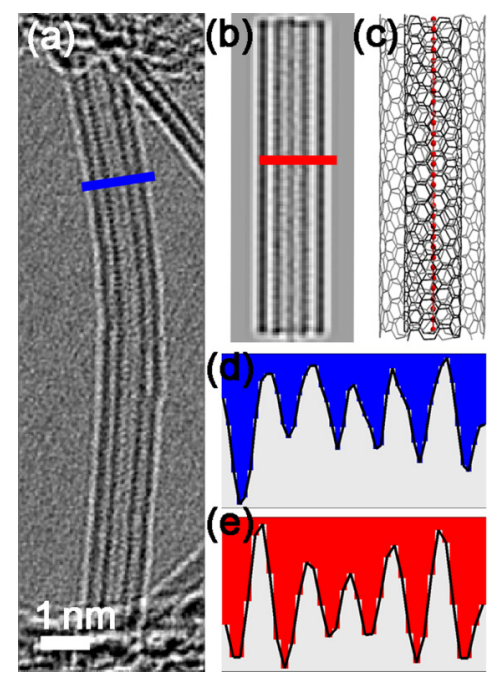

FIG. 1. An experimental HRTEM image (a), a simulated HRTEM image (b), and a molecular model (c) of a LLCC@DWCNT. The line profiles for the experimental (d) and the simulated (e) LLCC@DWCNT at the corresponding marked positions are shown in blue (d) and red (e). covered by strong CNT absorption peaks [23]. Therefore, we applied resonant Raman excitation spectroscopy to obtain the lowest singlet excitation energy (i.e., the optical band gap) of LLCC@DWCNT. The experiments were performed under ambient conditions using triple monochromator Raman spectrometers (Dilor XY in Vienna, Dilor XY800 in Antwerp, and Horiba T64000 in Berlin) in combination with several tunable laser systems. Dye lasers with Rhodamine 110, Rhodamine 6G, and DCM are used to tune the laser wavelength from 540 to $570 \mathrm{~nm}, 560$ to $620 \mathrm{~nm}$, and 620 to $680 \mathrm{~nm}$, respectively. A Ti:sapphire laser was also used to get the laser wavelengths between 680 and $770 \mathrm{~nm}$.

Calculations were performed for H-terminated polyynes with 12-102 carbon atoms obtained using the second-order approximate coupled-cluster (CC2) method. This method is a size-consistent coupled-cluster approximation that avoids delocalization problems of density-functional theory in extended systems. Our calculations are based on geometries optimized with SCS-MP2, which yields a similar BLA as the highly accurate CCSD(T) method [22]. The cc-pVDZ basis set and the turbomole software were used.

\section{RESULTS AND DISCUSSION}

A typical Raman spectrum of LLCCs@DWCNTs consists of the Raman responses from the DWCNTs and the encapsulated LLCCs in the region at around $1850 \mathrm{~cm}^{-1}$ [shown for $568 \mathrm{~nm}$ laser excitation in Fig. 2(a)]. The line-shape analysis of the LLCC band measured for different excitation wavelengths obtained at room temperature (Fig. 3) and at an excitation wavelength of $590 \mathrm{~nm}$ at low temperature [Fig. 2(b)] reveals that it consists of six clearly resolved Raman peaks. Each of these different LLCC Raman frequencies corresponds to a resonance at a different excitation wavelength, hence corresponding to LLCCs with different band gaps. The observation of these different band gaps and Raman frequencies can either be attributed to LLCCs with different lengths [22] and/or due to different environmental interactions [6,22]. Indeed, Fig. 2(b) shows that the radial breathing modes (RBMs) of the $(6,5)$ and $(6,4)$ inner tubes of LCC-filled DWCNTs are blueshifted with respect to the RBMs of freestanding DWCNTs, and both chiralities show a clearly different blueshift, indicating a different steric interaction between the chains and the tubes (similar to that observed for water-filling [24]). Such van der Waals interactions, and also other interactions such as charge transfer between the LCCs and host CNTs [22], influence the BLA of the encapsulated chains and hence will result in different observed Raman frequencies (and as we demonstrate in this work, different band gaps).

The fact that we observe a discrete set of Raman features can be explained by the fact that only a limited number of possible inner tube diameters are available and are suitable for LCC synthesis (only in the smallest diameters the ultralong chains are stable [6]). This number is larger than 6 , but the diameter of these inner tubes is distributed nonuniformly, and the linewidth of the LCC peaks is about two to three times broader than the LCC peak from an individual LCC@DWCNT obtained in near-field Raman spectra [6,25]. This leads us to conclude that there are a few components in each of the six peaks observed. 

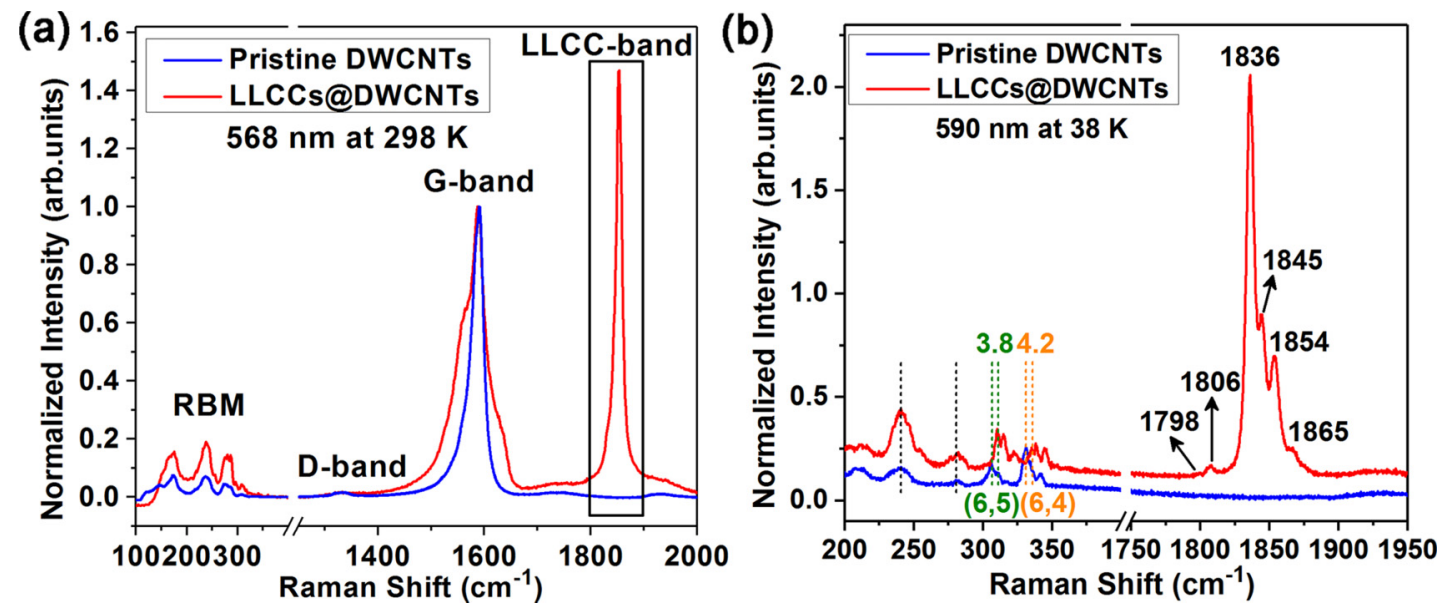

FIG. 2. (a) Raman spectra of pristine DWCNTs (blue line) and LLCC@DWCNTs (red line) measured with a $568 \mathrm{~nm}$ laser excitation at room temperature. The LLCC-band region is highlighted by a box. (b) Raman spectra of the same samples conducted with a $590 \mathrm{~nm}$ laser at $38 \mathrm{~K}$. The colored dashed lines indicate the blueshift of 4.2 and $3.8 \mathrm{~cm}^{-1}$ for the RBM peaks of $(6,4)$ and $(6,5)$ tubes, respectively, after encapsulation of the LLCCs. The black numbers are the frequencies of all observed Raman bands of the LLCCs.

Following the general trend that the longer the LCC is, the smaller the BLA and the resulting energy gap becomes, resonant Raman scattering is the ideal technique to identify the energy gap of ultralong LCCs encapsulated within the DWCNTs. Figure 3 shows the Raman spectra as a function of laser excitation wavelength for different excitation ranges. For each of these excitation wavelengths (more than 50), we extracted the Raman intensities of the six LLCC bands that are resolved. The resonance Raman excitation profiles obtained are presented in Fig. 4. Note that two of the LLCC bands correspond to Raman frequencies around $1800 \mathrm{~cm}^{-1}$, as we found previously for chains with lengths of several thousands of carbon atoms in near-field Raman spectroscopy [6], sufficiently long for the LCC properties to have converged to those of infinitely long LCCs (carbyne).

The analysis of the energy gaps from the resonance Raman excitation profiles was performed by fitting the separate Raman peaks in the LLCC band and plotting their intensities as a function of excitation energy. The profiles for six LLCC
Raman peaks are shown in Fig. 4(a) with excitation energy steps smaller than $0.007 \mathrm{eV}$. These six profiles indicate strong electronic resonances that peak at different energy for each group of LCCs, which matches the electronic energy gap of LCCs. The profiles can be fitted by a semiclassical resonance Raman model [27-29]:

$$
I\left(E_{L}\right) \propto\left|\frac{M}{E_{L}-E_{\mathrm{op}}+i \frac{\Gamma}{2}}\right|^{2},
$$

where $M, E_{L}, E_{\mathrm{op}}$, and $\Gamma$ are the incident resonance factor, laser excitation, optical transition, and an electronic broadening term, respectively. Note that a quantum model should be applied when considering both the incident and scattered resonances [26]. This is not the case here, since we only measured the incident resonance. Applying Eq. (1) to fit the experimental profiles was done by adjusting $M, E_{\mathrm{op}}$, and $\Gamma$. The obtained $E_{\mathrm{op}}$ and $\Gamma$ are summarized in Table I. The widths of the excitation profiles are similar to the width of RBM or
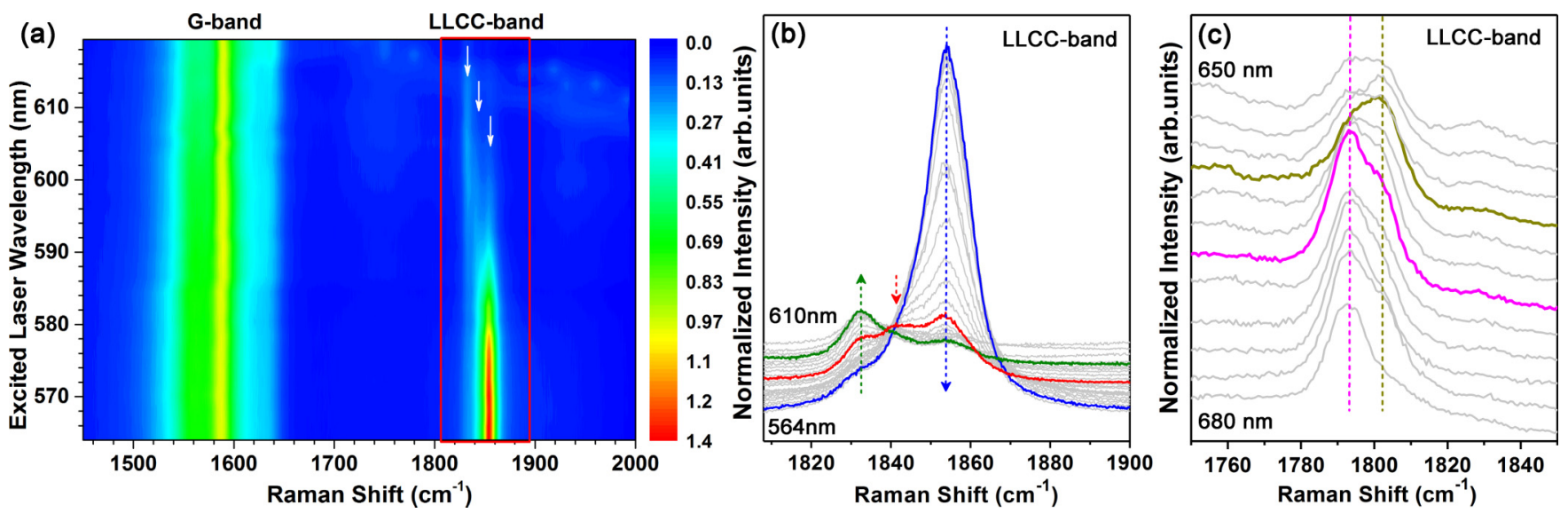

FIG. 3. (a) Resonance Raman mapping of LLCC@DWCNTs. The LLCC band is highlighted by a red box. The three arrows point out three LLCC-band peaks with different frequencies. The evolution of the Raman spectra of LLCC@DWCNTs excited by lasers with a wavelength 564-610 nm (b) and 650-680 nm (c). The resonance Raman spectra for the LLCC peaks at 1793, 1802, 1832, 1842, as well as the sum of 1850 and $1856 \mathrm{~cm}^{-1}$ were highlighted by magenta, dark yellow, olive, red, and blue lines, respectively. 


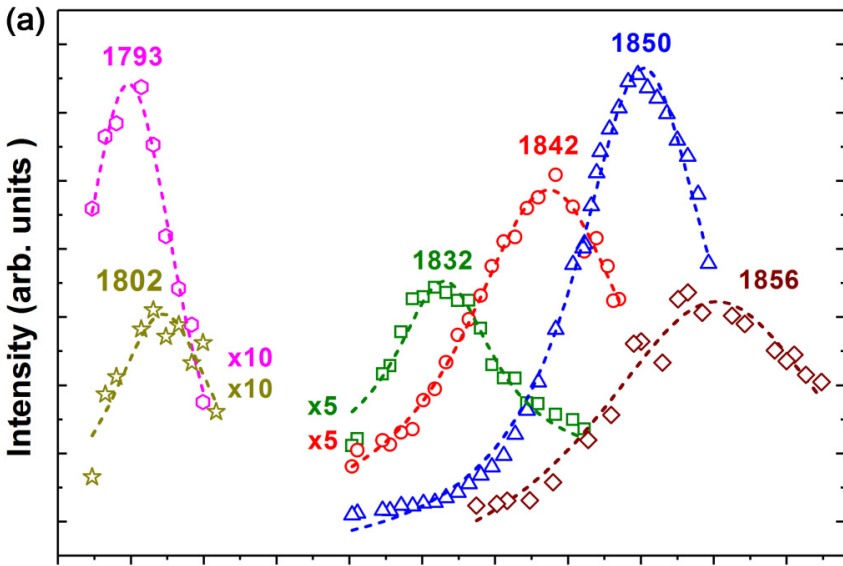

$\begin{array}{lllllllllll}1.80 & 1.85 & 1.90 & 1.95 & 2.00 & 2.05 & 2.10 & 2.15 & 2.20 & 2.25 & 2.30\end{array}$ Laser Energy (eV)

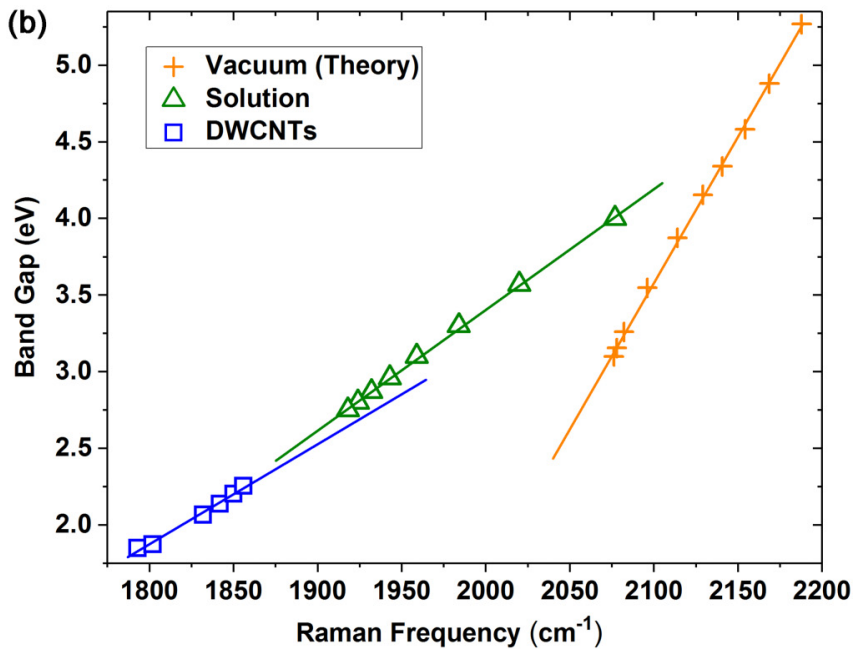

FIG. 4. (a) Resonance Raman excitation profiles for six LLCCband peaks. The dashed lines are a fit to the experimental data using Eq. (1). (b) Band gap of the LCCs as a function of Raman frequency of the LCCs. The orange crosses are our theoretical prediction by $a b$ initio calculations on the free chains in vacuum, the olive triangles represent LCCs terminated by bulky end groups in toluene (Raman frequencies) or hexane (band gap) [4,5], and the blue squares are our work on LCCs inside DWCNTs. The linear lines are the fittings of the data points.

G-band excitation profiles in SWCNTs [27-29]. The width of the peak located at $1856 \mathrm{~cm}^{-1}$ is wider than the widths of the other peaks, indicating that the $1856 \mathrm{~cm}^{-1}$ peak includes even more components than the others.

$E_{\text {op }}$ gives the band gaps. Figure 4(b) plots the band gap of the six resolved components as a function of Raman frequency (blue squares), and it compares this with previous experimental data of short LCCs obtained in solution and with our own theoretical calculations. All three datasets show a

TABLE I. Fitting analysis of resonance Raman excitation profiles.

\begin{tabular}{lcccccc}
\hline \hline Frequency $\left(\mathrm{cm}^{-1}\right)$ & 1793 & 1802 & 1832 & 1842 & 1850 & 1856 \\
\hline$E_{\text {op }}(\mathrm{eV})$ & 1.848 & 1.872 & 2.065 & 2.137 & 2.202 & 2.253 \\
$\Gamma(\mathrm{meV})$ & 72 & 84 & 116 & 131 & 97 & 145 \\
\hline \hline
\end{tabular}

remarkably accurate linear dependence of the band gap on the Raman frequency, albeit with a different slope, demonstrating that the band gap is modulated in the same manner as the Raman frequency through the BLA [22]. Such linear relations between excitation energy, BLA, and vibrational frequencies are also known from other systems exposed to noncovalent interactions, e.g., the retinal chromophore inside different rhodopsin proteins [30,31]. Note that for the solution data presented in Fig. 4(b), optical band gaps were determined previously in hexane while Raman frequencies of the same chains were obtained in toluene, hence a different environment. The data for the encapsulated chains inside DWCNTs and the solution data were therefore fitted separately.

It is remarkable that the accurate linear relation between the Raman frequency and the band gap holds over such a wide range, considering that many different factors are expected to lie at the origin of the variation of both. In general, the Raman frequency and the band gap are directly related to the BLA arising from the Peierls distortion of the LCC [14]. Indeed, previous theoretical and experimental work demonstrated that by applying strain to deliberately change the length of the carbon bonds, cumulene can be tuned from metallic $(\mathrm{BLA}=0)$ to semiconducting $(\mathrm{BLA}>0)$, and finally it becomes insulating [32-34]. Similarly, the band gap of graphene or other 2D materials can be adjusted by strain, type of stacking, charging the substrate, chemical functionalization, electronic doping, etc. [9-12]. Previous studies have demonstrated that the BLA not only depends on the intrinsic length of the LCCs [3], but also on extrinsic factors such as environment interactions (van der Waals interactions, charge transfer, and dielectric screening) $[3,18,22,35]$ and the specific choice of chemical groups at the end of the chains $[5,15]$. The relative contribution of each of these effects in our experimental data is, however, difficult to disentangle and will be different for different length ranges. For short chains, with lengths ranging from 6 to 44 carbon atoms, as previously measured in solution $[5,15]$ or in the gas phase [36], it is well known that the length of the chains strongly influences the BLA and hence the Raman frequency [22]. Indeed, when plotting the experimentally determined band gaps of those chains with well-defined lengths as a function of the inverse number of carbon atoms, a linear dependence on $1 / N$ is obtained, with $N$ the number of carbon atoms in the chain (solid triangles, squares, and circles in Fig. 5). To extend the band-gap dependence on length to longer chain lengths than those measured in the gas phase, we also included our calculated excitation energies of $\mathrm{H}$-terminated polyynes with 12-102 carbon atoms (orange crosses in Fig. 5). The excitation energies for the lowest allowed singlet transition, as presented in Fig. 5, agree very well with the gas-phase measurements [36], and they show a deviation from the linear dependence for the longest chains, approaching the band gap of $3.3 \mathrm{eV}$ of carbyne obtained from the diffusion Monte Carlo result of Mostaani et al. [13]. Interestingly, the LCCs measured in solution show a significant downshift of the band-gap energy with respect to the gas-phase measurements, indicating that the band gap is very sensitive to the environment through van der Waals interaction, dielectric screening, and/or a charge-transfer interaction. In addition, the groups that are at the end of the chains (end caps) also influence the BLA. 


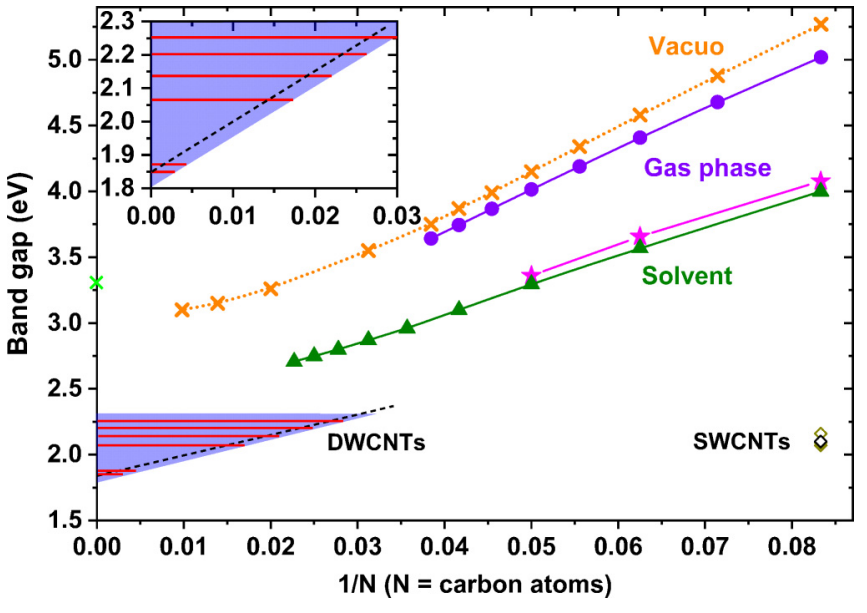

FIG. 5. The band gap as a function of the inverse number of carbon atoms by ab initio calculations (our work: orange crosses), predicted by Mostaani et al. for carbyne (green cross at $1 / N=0$ ) [13], measured in gas phase (solid circles) [36] or dissolved in a solvent (solid triangles and solid stars represent LCCs terminated by different chemical ending groups) [5,15] by absorption spectroscopy, and LCCs inside SWCNTs (open squares [18,35])/DWCNTs (our work: the light blue shadow) by resonance Raman spectroscopy. The data for SWCNTs do not represent band-gap measurements but excited dark states [18,35]. Inset: the enlarged part of the band gap of the confined chains. The dashed line is obtained by shifting the linear fit of the LCCs in solution, with the smallest observed band gap as the anchor point. The horizontal red lines represent the measured band gaps, and the blue shaded area indicates the possible length range for our data.

As shown in Fig. 5, the solid triangles and stars represent chains that are terminated with different end groups while surrounded by the same solvent, resulting in a shift of about $0.1 \mathrm{eV}[5,15]$. In particular, for short chains one can expect a strong influence of these end groups on the band gap of the chains [37], which can be used to tune this band gap to some extent [38]. Implementing our measured band gaps into Fig. 5 (red horizontal lines represent the measured band gaps) is not so straightforward, as the actual lengths of the LCCs in our samples vary from 30 up to more than 6000 atoms [6]. From near-field Raman spectroscopy, it is known that at least for LCCs with lengths longer than $30 \mathrm{~nm}$ (i.e., $N>230$ ) [25], the interactions with the environment (i.e., the chirality of the surrounding inner CNT) dominate over length in determining the vibrational frequency.

Combining this information for short and long chains, we can define the upper and lower bounds to the lengths of the LCCs in our experimental data. If, in one limiting case, we assume that all the Raman frequencies in our experiments originate from chains longer than $30 \mathrm{~nm}(1 / N$ approaching 0$)$, and hence the length hardly influences the Raman frequency, then the six different Raman peaks that are observed can be attributed to interactions with different inner tube chiralities. However, if we assume that also shorter chains contribute to the observed Raman frequencies, as these are also present in our samples [6], then the length can also contribute significantly to the observed band-gap variations. To define the upper limit in $1 / N$, one can assume all chains encapsulated in the same CNT environment, i.e., the one that yields the largest possible downshift. Then the observed band gaps would depend solely on the chain length. Hence we take a similar length dependence as obtained for the short chains in solution (i.e., similar slope), but we shift it to lower energy with the lowest measured band gap as the anchor point for the longest chains (dashed line in Fig. 5). Note that the shift to lower energy for chains encapsulated in DWCNTs can be explained in the same manner as the shift from gas phase to solution spectra. Most likely, in our samples including both shorter and ultralong chains, chain lengths are in between these two limits (augmented with an experimental error margin), as highlighted by the shaded area in Fig. 5. If the exact curve saturates for large $N$, like our theoretical gas-phase results, the shaded area would extend further to the right, but the limiting values for the band gap of confined carbyne would be unaffected. The smallest band gap measured in our samples is $1.848 \mathrm{eV}$ and is much lower than the value of the band gap of carbyne $(2.56 \mathrm{eV})$, which was extrapolated from the band gap of short chains with lengths only ranging from 6 to 44 carbon atoms [4,5]. However, our results demonstrate that such an extrapolation is difficult to perform, as for long chains the length is no longer a determining factor for the band gap, and in particular also the interaction with the environment needs to be taken into account.

Our experimental data cannot resolve which interaction dominates. The chirality-dependent blueshift of the RBMs of LCC-filled CNTs with respect to pristine CNTs [Fig. 2(b)] suggests a steric interaction between the LCCs and the CNTs, similar as observed previously for water-filling [24,39]. Previous experimental studies also reported that the band gap of the LCC inside MWCNTs does not depend on the number of host CNT walls [16,40,41], rather it depends on the diameter of the innermost tubes. In addition, when encapsulating the same $\mathrm{C}_{10} \mathrm{H}_{2}$ chain inside three different SWCNT diameter distributions, an energy shift of the order of $0.1 \mathrm{eV}$ was observed as a consequence of different dielectric screening [18]. Indeed, a recent theoretical work showed that, apart from charge transfer [3], van der Waals interactions strongly affect the electronic structure, BLA, and vibrational properties of encapsulated polyynes [22]. Overall, it is still a big challenge to quantitatively evaluate the effects of van der Waals interaction, dielectric screening, or charge transfer on the hybrid LCC@CNT system theoretically or experimentally.

\section{CONCLUSIONS}

In summary, the band gaps of confined LCCs were directly measured by resonance Raman excitation spectroscopy. An accurate linear relation between Raman frequency and band gap was obtained. The LCCs inside DWCNTs possess band gaps of 2.253-1.848 eV. The band gap of $1.848 \mathrm{eV}$ for the long confined LCCs is the smallest band gap observed so far. Note that the band-gap values reported here are the optical band gaps, and thus they include (reduction by) the exciton binding energy, as is the case also in all previous measurements on short chains. Theoretical calculations show that the exciton binding energy of carbon chains is rather small (about $0.1 \mathrm{eV}$ ) [13]. Our results illustrate the theoretical challenges of taking into account the interactions with the environment to calculate the band gap of LCCs. LCCs were predicted to be the stiffest materials [32], and they can even be used for spin transport 
[42]. Also, the LCC@CNT system can achieve metallic transport properties by a high density of states at the Fermi level, due to a combined effect of orbital hybridization and charge transfer $[43,44]$. Together with the tunable band gap, LCCs would be a promising candidate for future nanoelectronic, photonic, and spintronic devices.

\section{ACKNOWLEDGMENTS}

This work was supported by the Austrian Science Funds (FWF, P27769-N20) and the EU project (2D-Ink FA726006). L.S. gratefully acknowledges the scholarship supported by the
China Scholarship Council. A.R. acknowledges financial support from the European Research Council (ERC-2015-AdG694097), Grupos Consolidados (IT578-13), and NOMAD (GA No. 676580). P.R., S.C., and W.W. acknowledge funding from the Fund for Scientific Research Flanders, Belgium (FWO, projects No. G040011N, No. G021112N, No. 1513513N, and No. $1512716 \mathrm{~N}$ ), which also supported S.C. through a postdoctoral fellowship. S.C. also acknowledges funding from European Research Council Starting Grant No. ERC-2015StG-679841. We thank Kazu Suenaga and Yoshiko Niimi for the HRTEM measurements, Hans Kuzmany for insightful discussion, and Patryk Kusch for Ti:sapphire laser preparation.
[1] M. Kertesz, J. Koller, and A. Azman, J. Chem. Phys. 68, 2779 (1978).

[2] C. S. Casari, M. Tommasini, R. Tykwinski, and A. Milani, Nanoscale 8, 4414 (2016).

[3] S. Yang and M. Kertesz, J. Phys. Chem. A 110, 9771 (2006).

[4] W. A. Chalifoux and R. R. Tykwinski, Nat. Chem. 2, 967 (2010).

[5] N. R. Agarwal, A. Lucotti, D. Fazzi, M. Tommasini, C. Castiglioni, W. A. Chalifoux, and R. R. Tykwinski, J. Raman Spectrosc. 44, 1398 (2013).

[6] L. Shi, P. Rohringer, K. Suenaga, Y. Niimi, J. Kotakoski, J. C. Meyer, H. Peterlik, M. Wanko, S. Cahangirov, A. Rubio, Z. J. Lapin, L. Novotny, P. Ayala, and T. Pichler, Nat. Mater. 15, 634 (2016).

[7] Carbon Nanotubes: Advanced Topics in the Synthesis, Structure, Properties, and Applications, edited by A. Jorio, G. Dresselhaus, and M. S. Dresselhaus (Springer-Verlag, Berlin, Heidelberg, 2008).

[8] C. Fantini, A. Jorio, M. Souza, M. S. Strano, M. S. Dresselhaus, and M. A. Pimenta, Phys. Rev. Lett. 93, 147406 (2004).

[9] A. C. Neto, F. Guinea, N. Peres, K. S. Novoselov, and A. K. Geim, Rev. Mod. Phys. 81, 109 (2009).

[10] Q. H. Wang, K. Kalantar-Zadeh, A. Kis, J. N. Coleman, and M. S. Strano, Nat. Nano. 7, 699 (2012).

[11] V. Tran, R. Soklaski, Y. Liang, and L. Yang, Phys. Rev. B 89, 235319 (2014).

[12] G. E. Eperon, S. D. Stranks, C. Menelaou, M. B. Johnston, L. M. Herz, and H. J. Snaith, Energy Environ. Sci. 7, 982 (2014).

[13] E. Mostaani, B. Monserrat, N. Drummond, and C. Lambert, Phys. Chem. Chem. Phys. 18, 14810 (2016).

[14] R. E. Peierls, Quantum Theory of Solids (Oxford University Press, Oxford, 1955).

[15] S. Eisler, A. D. Slepkov, E. Elliott, T. Luu, R. McDonald, F. A. Hegmann, and R. R. Tykwinski, J. Am. Chem. Soc. 127, 2666 (2005).

[16] C. Fantini, E. Cruz, A. Jorio, M. Terrones, H. Terrones, G. Van Lier, J. C. Charlier, M. S. Dresselhaus, R. Saito, Y. A. Kim, T. Hayashi, H. Muramatsu, M. Endo, and M. A. Pimenta, Phys. Rev. B 73, 193408 (2006).

[17] L. M. Malard, D. Nishide, L. G. Dias, R. B. Capaz, A. P. Gomes, A. Jorio, C. A. Achete, R. Saito, Y. Achiba, H. Shinohara, and M. A. Pimenta, Phys. Rev. B 76, 233412 (2007).

[18] L. G. Moura, L. M. Malard, M. A. Carneiro, P. Venezuela, R. B. Capaz, D. Nishide, Y. Achiba, H. Shinohara, and M. A. Pimenta, Phys. Rev. B 80, 161401 (2009).
[19] N. Andrade, T. Vasconcelos, C. Gouvea, B. Archanjo, C. Achete, Y. Kim, M. Endo, C. Fantini, M. Dresselhaus, and A. Souza Filho, Carbon 90, 172 (2015).

[20] C.-S. Kang, K. Fujisawa, Y. Ko, H. Muramatsu, T. Hayashi, M. Endo, H. J. Kim, D. Lim, J. H. Kim, Y. C. Jung, M. Terrones, M. S. Dresselhaus, and Y. A. Kim, Carbon 107, 217 (2016).

[21] T. Hayashi, H. Muramatsu, Y. A. Kim, H. Kajitani, S. Imai, H. Kawakami, M. Kobayashi, T. Matoba, M. Endo, and M. S. Dresselhaus, Carbon 44, 1130 (2006).

[22] M. Wanko, S. Cahangirov, L. Shi, P. Rohringer, Z. J. Lapin, L. Novotny, P. Ayala, T. Pichler, and A. Rubio, Phys. Rev. B 94, 195422 (2016).

[23] P. Rohringer, L. Shi, P. Ayala, and T. Pichler, Adv. Funct. Mater 26, 4874 (2016).

[24] S. Cambré, B. Schoeters, S. Luyckx, E. Goovaerts, and W. Wenseleers, Phys. Rev. Lett. 104, 207401 (2010).

[25] S.. Heeg, L. Shi, L. V. Poulikakos, T. Pichler, and L. Novotny, arXiv:1711.04753.

[26] W. L. Peticolas, L. Nafie, P. Stein, and B. Fanconi, J. Chem. Phys. 52, 1576 (1970).

[27] J. G. Duque, H. Chen, A. K. Swan, A. P. Shreve, S. Kilina, S. Tretiak, X. Tu, M. Zheng, and S. K. Doorn, ACS Nano 5, 5233 (2011).

[28] E. H. Hároz, J. G. Duque, E. B. Barros, H. Telg, J. R. Simpson, A. R. Hight Walker, C. Y. Khripin, J. A. Fagan, X. Tu, M. Zheng, J. Kono, and S. K. Doorn, Phys. Rev. B 91, 205446 (2015).

[29] H. N. Tran, J.-C. Blancon, J.-R. Huntzinger, R. Arenal, V. N. Popov, A. A. Zahab, A. Ayari, A. San-Miguel, F. Vallée, N. Del Fatti, J. L. Sauvajol, and M. Paillet, Phys. Rev. B 94, 075430 (2016).

[30] G. Kochendoerfer, Z. Wang, D. D. Oprian, and R. A. Mathies, Biochem. 36, 6577 (1997).

[31] J. S. Frähmcke, M. Wanko, and M. Elstner, J. Phys. Chem. B 116, 3313 (2012).

[32] M. Liu, V. I. Artyukhov, H. Lee, F. Xu, and B. I. Yakobson, ACS Nano 7, 10075 (2013).

[33] V. I. Artyukhov, M. Liu, and B. I. Yakobson, Nano Lett. 14, 4224 (2014).

[34] A. La Torre, A. Botello-Mendez, W. Baaziz, J.-C. Charlier, and F. Banhart, Nat. Commun. 6, 6636 (2015).

[35] D. Nishide, T. Wakabayashi, T. Sugai, R. Kitaura, H. Kataura, Y. Achiba, and H. Shinohara, J. Phys. Chem. C 111, 5178 (2007).

[36] T. Pino, H. Ding, F. Güthe, and J. P. Maier, J. Chem. Phys. 114, 2208 (2001). 
[37] M. Weimer, W. Hieringer, F. Della Sala, and A. Görling, Chem. Phys. 309, 77 (2005).

[38] A. Milani, M. Tommasini, V. Barbieri, A. Lucotti, V. Russo, F. Cataldo, and C. S. Casari, J. Phys. Chem. C 121, 10562 (2017).

[39] W. Wenseleers, S. Cambré, J. Čulin, A. Bouwen, and E. Goovaerts, Adv. Mater. 19, 2274 (2007).

[40] X. L. Zhao, Y. Ando, Y. Liu, M. Jinno, and T. Suzuki, Phys. Rev. Lett. 90, 187401 (2003).
[41] L. Shi, L. M. Sheng, L. M. Yu, K. An, Y. Ando, and X. L. Zhao, Nano Res. 4, 759 (2011).

[42] Z. Zanolli, G. Onida, and J. C. Charlier, ACS Nano 4, 5174 (2010).

[43] Á. Rusznyak, V. Zólyomi, J. Kürti, S. Yang, and M. Kertesz, Phys. Rev. B 72, 155420 (2005).

[44] A. Tapia, L. Aguilera, C. Cab, R. A. Medina-Esquivel, R. de Coss, and G. Canto, Carbon 48, 4057 (2010). 